\title{
跨膜 $\mathrm{Ca}^{2+}$ 梯差对重建 $\boldsymbol{\beta}$-肾上腺素能 受体配基结合功能的影响
}

\author{
杨小媇 范高峰 黄有国 杨福愉 * \\ (生物大分子国家重点实验室, 中国科学院生物物理研究所, 北京 100101)
}

\section{关键词跨膜 $\mathrm{C}^{2+}$ 梯差 $\mathrm{P}-$ 肾上腺素能受体 配基结合}

在正常生理状态下, 真核细胞内 $\mathrm{Ca}^{2+}$ 浓度为 $10^{-7} \sim 10^{-6} \mathrm{~mol} / \mathrm{L}$, 细胞外侧为 $10^{-3} \mathrm{~mol} / \mathrm{L}$, 即细胞膜的两侧存在 $1000 \sim 10000$ 倍的跨膜 $\mathrm{Ca}^{2+}$ 梯差. 当细胞外信息跨膜传递时细胞外 $\mathrm{Ca}^{2+}$ 内流, 胞浆中的 $\mathrm{Ca}^{2+}$ 浓度升高, 细胞膜两侧的跨膜 $\mathrm{Ca}^{2+}$ 梯差降低约 10 倍, 即膜内外两侧 的跨膜 $\mathrm{Ca}^{2+}$ 梯差为 100 倍 ${ }^{[1]}$; 而信息传递完成后胞浆中的 $\mathrm{Ca}^{2+}$ 会通过质膜上的 $\mathrm{Ca}^{2+}$-ATP 酶 或 $\mathrm{Na}^{+}-\mathrm{Ca}^{2+}$ 交换运出细胞外以维持膜两侧合适的跨膜 $\mathrm{Ca}^{2+}$ 梯差. 因此, 细胞膜两侧的跨膜 $\mathrm{Ca}^{2+}$ 梯差在维持细胞正常功能中具有重要的生理意义 ${ }^{[2]}$. 但这种跨膜 $\mathrm{Ca}^{2+}$ 梯差对膜结合蛋 白, 尤其是对参与构成信息跨膜转导体系的膜蛋白的结构与功能及其相互作用的影响尚未引 起足够的重视.

cAMP 体系是目前已知的 5 种重要的信息跨膜转导体系之一, 它是由膜上的受体、激活型 $\mathrm{G}$ 蛋白 (Gs-protein)和效应器腺苷酸环化酶 (Adenylyl cyclase, AC) 构成. 肾上腺素、去甲肾上 腺素和异丙肾上腺素等配基与 $\beta$-肾上腺素能受体 ( $\beta$-Adrenergic receptor, $\beta$-AR)结.合后, 通过 定位于细胞膜内侧的激活型 Gs 的偶联, 激活 $\mathrm{AC}$ 活力, 催化细胞内第二信使 cAMP 的形成, 从 而调节细胞的生长、增殖、分化及调亡等功能活动 ${ }^{[3]}$. 因此, 配基识别与结合是启动信息跨膜 转导反应的最初事件, 对这种起始反应的调节因素的研究, 对于阐明信息跨膜转导的分子机理 具有极为重要的生物学意义. 本文应用生物膜的拆离与重建技术, 从北京鸭红细胞中纯化得 到 $\beta$-AR, 并将其重建于大豆磷脂脂质体, 形成由外向内为 $1 \sim 1000$ 倍的不同跨膜 $\mathrm{Ca}^{2+}$ 浓度梯 差的 3 种脂蛋白体 $L \cdot(\beta-A R)$, 以研究跨膜 $\mathrm{Ca}^{2+}$ 梯差对重建 $\beta-A R$ 的配基结合功能的影响.

\section{1 材料与方法}

\section{1 材料}

北京鸭购自北京玉泉鸭厂; ( \pm ) Alprenolol, ( - ) Isoproterenol, Digitonin, PMSF、正辛基 葡萄糖式和大豆磷脂均为 Sigma 产品; Sepharose 4B 为 Pharmacia 产品; Alprenolol agarose gel 购自 ICN 公司; $\left[{ }^{3} \mathrm{H}\right]-\mathrm{DHA}$ ( $\left[{ }^{3} \mathrm{H}\right]$-二氢心得舒) 为 DuPont 公司产品.

1995-08-08 收稿, 1995-12-06 收修改稿

* 联系人 


\section{2 方法}

\subsection{1 北京鸭红细胞膜制备 参照 $\Phi$ ye 和 Sutherland ${ }^{[4]}$ 的方法.}

1.2.2 B-肾上腺素能受体的分离纯化参照 Caron 等 ${ }^{[5]}$ 和范高峰等 ${ }^{[6]}$ 改进的亲和色谱方 法, 以 $1 \%$ Digitonin 增溶鸭红细胞膜, 将增溶物经两次心得舒-Agarose 亲和层析柱进一步纯 化. 所提纯的蛋白经 SDS-PAGE 鉴定为 $\beta_{1}-A R$, 分子量为 68000 .

1.2.3 $\beta$-肾上腺素能受体在具有不同 $\mathrm{Ca}^{2+}$ 梯差的大豆磷脂脂质体上的重建 ${ }^{[6]}$ 大豆磷脂 $7.5 \mathrm{mg}$ 分别悬浮于含不同浓度 $\mathrm{Ca}^{2+}$ 的 $10 \mathrm{mmol} / \mathrm{L}$ Tris- $\mathrm{HCl}$ 缓冲液 $(\mathrm{pH} 7.6$ ) 和 $0.85 \%$ 正辛基 葡萄糖式溶液中, 超声至清亮, 与 $0.65 \mathrm{nmol} \beta-\mathrm{AR}$ 混合, $0^{\circ} \mathrm{C}$ 孵育 $30 \mathrm{~min}$ 后, 上样于 Sepharose $4 \mathrm{~B}$ 色谱柱 $(5 \mathrm{~mL}$ ), 用上述缓冲液洗脱, 收集洗脱峰, 离心 $60000 \times g 30 \mathrm{~min}$, 沉淀 (脂蛋白体) 悬浮于 $1.0 \mathrm{~mL}$ 含 $1 \mathrm{mmol} / \mathrm{L} \mathrm{Ca}^{2+}$ 的上述 Tris- $\mathrm{HCl}$ 缓冲液中即脂蛋白体外 $\left[\mathrm{Ca}^{2+}\right]$ 为 $1 \mathrm{mmol} /$ $\mathrm{L}$, 获得含 $\beta-A R$ 的具有不同 $\mathrm{Ca}^{2+}$ 梯差的脂蛋白体, 即:

脂蛋白体 $\mathrm{A}:$ 脂蛋白体内为 $1 \mu \mathrm{mol} / \mathrm{L} \mathrm{Ca}^{2+},\left[\mathrm{Ca}^{2+}\right]_{\mathrm{O}}:\left[\mathrm{Ca}^{2+}\right]_{\mathrm{i}}=1000: 1$, 类似于生理条 件;

脂蛋白体 $\mathrm{B}$ : 脂蛋白体内为 $10 \mu \mathrm{mol} / \mathrm{L} \mathrm{Ca}^{2+},\left[\mathrm{Ca}^{2+}\right]_{\mathrm{O}}:\left[\mathrm{Ca}^{2+}\right]_{\mathrm{i}}=1000: 10$, 类似于细胞外 $\mathrm{Ca}^{2+}$ 内流后的跨膜 $\mathrm{Ca}^{2+}$ 梯差;

脂蛋白体 C: 脂蛋白体内为 $1 \mathrm{mmol} / \mathrm{L} \mathrm{Ca}^{2+},\left[\mathrm{Ca}^{2+}\right]_{\mathrm{O}}:\left[\mathrm{Ca}^{2+}\right]_{\mathrm{i}}=1000: 1000$, 无跨膜 $\mathrm{Ca}^{2+}$ 梯差.

1.2.4 $\beta$-肾上腺素能受体的放射性配基 $\left(\left[{ }^{3} \mathbf{H}\right]\right.$-DHA $)$ 结合测定 参照 Vauquelin 等 ${ }^{[7]}$ 的方 法, 用不同浓度的 $\left[{ }^{3} \mathrm{H}\right]-\mathrm{DHA}$ 分别与上述含 $\beta$-AR 的具有不同 $\mathrm{Ca}^{2+}$ 梯差的脂蛋白体混匀, 反应 总体积 $220 \mu \mathrm{L}, 30^{\circ} \mathrm{C}$ 餒育 $10 \mathrm{~min}$ 后, 立即置于冰浴终止反应, 真空抽滤, 用 $75 \mathrm{mmol} / \mathrm{L}$ Tris$\mathrm{HCl}$ 缓冲液 ( $\mathrm{pH}$ 7.6) 冲洗滤膜 5 次, 烘干过夜后, 置于 $5 \mathrm{~mL}$ 甲苯闪栋液中, 用 Beckman LS9800 液闪计数仪测定滤膜的放射性, 计算样品与 $\left[{ }^{3} \mathrm{H}\right]-\mathrm{DHA}$ 的总结合量. 另在平行管中加 $入 10 \mu \mathrm{mol} / \mathrm{L}( \pm)$-Alprenolol, 测定 $\left[{ }^{3} \mathrm{H}\right]-\mathrm{DHA}$ 的非特异结合量, 两者之差为受体对 DHA 的特 异结合量. 以 $\left[{ }^{3} \mathrm{H}\right]$-DHA 特异结合量 $(B)$ 对其浓度 $(F)$ 作饱和曲线, 以 $B / F$ 对 $B$ 作 Scatchard 图, 直线斜率的负倒数为平衡解离常数 $K_{\mathrm{D}}$, 与 $x$ 轴的交点值为结合容量 $R$.

\subsection{5 蛋白质含量测定 蛋白质的含量采用 Lowry 法测定，}

\section{2 结果和讨论}

大约 $80 \%$ 的激素和神经递质激活细胞的信息跨膜机理都是通过激活偶联 $\mathrm{G}$ 蛋白的受体 而实现的 ${ }^{[8]}, \beta-A R$ 是一种与 Gs 蛋白紧密偶联的具有高度疏水性的跨膜蛋白, 由细胞外 N-末 端、7 个跨膜螺旋 (seven-transmembrane-segment, 7-TMS) 和细胞内 C-末端 3 个结构域组成. $\beta-A R$ 与配基(激动剂或拮抗剂)相结合的位点位于膜脂双层中心疏水区, 其 7-TMS 微区形成 袋状结合间隙 (binding pocket), $\beta$-AR 与配基的结合主要通过配基的氨基基团与受体疏水区的 羧基侧链之间形成盐桥的相互作用. 通常用配基结合容量(受体结合位点数目或受体密度 $R)$ 和亲和力 $\left(K_{\mathrm{D}}\right)$ 来评价受体的配基结合功能.

为了研究跨膜 $\mathrm{Ca}^{2+}$ 梯差对 $\beta-\mathrm{AR}$ 的配基结合功能的影响, 本文将纯化的 $\beta$-肾上腺素能受 体 ( $\beta-\mathrm{AR}$ ) 重建于大豆磷脂脂质体上, 并通过改变脂蛋白体内的 $\mathrm{Ca}^{2+}$ 浓度制备了含 $\beta-A R$ 的具 有不同 $\mathrm{Ca}^{2+}$ 梯差的 3 种脂蛋白体 $\mathrm{L} \cdot(\beta-\mathrm{AR})$, 并测定其受体对放射性配基 $\left[{ }^{3} \mathrm{H}\right]-\mathrm{DHA}$ 的结合 
活性. 图 1 示 3 种脂蛋白体中 $\beta-\mathrm{AR}$ 的配基结合饱和曲线, 可以看出, 不同跨膜 $\mathrm{Ca}^{2+}$ 梯差条件 下, 重建 $\beta-A R$ 与放射性配基的特异结合量随着配基 $\left[{ }^{3} \mathrm{H}\right]$-DHA 的量而增加, 呈现出典型的 $\mathrm{S}$ 型量-效反应曲线, 经 Scatchard 作图, 反应曲线表现为向下凹陷的曲线 (图 2), 可将其分解为两 条不同的直线. 这一结果与本实验室有关北京鸭红细胞膜 $\beta-A R$ 可能含有两种不同的结合位 点即高亲和力/低结合容量结合位点和低亲和力/高结合容量结合位点的结果相一致(另文报 道). 进一步分析跨膜 $\mathrm{Ca}^{2+}$ 梯差对重建 $\beta-\mathrm{AR}$ 的配基结合功能的影响(表 1), 在不同跨膜 $\mathrm{Ca}^{2+}$ 梯差条件下, 不论是高亲和力的结合位点还是低亲和力的结合位点, 跨膜 $\mathrm{Ca}^{2+}$ 梯差对二者平 衡解离常数即受体与配基结合的亲和力的影响均是一致的, $K_{\mathrm{D} 1}$ 和 $K_{\mathrm{D} 2}$ 的大小均为 $A<B<$ $C$, 即亲和力的大小为 $A>B>C$, 说明一个与生理状态相似的跨膜 $\mathrm{Ca}^{2+}$ 梯差对 $\beta-A R$ 与配基 的牢固结合是必需的. 但从跨膜 $\mathrm{Ca}^{2+}$ 梯差对受体结合容量的影响来分析, 由计算机拟合得 出, $27.55 \mathrm{nmol} / \mathrm{L}$ 是其结合反应的拐点 (见图 1 的 “ $\rightarrow$ ”所示, 即 1.44 的反对数), 在 $\left[{ }^{3} \mathrm{H}\right]-\mathrm{DHA}$ 浓度小于 $27.55 \mathrm{nmol} / \mathrm{L}$ 时, 重建脂蛋白体 $\beta-\mathrm{AR}$ 与 $\left[{ }^{3} \mathrm{H}\right]-\mathrm{DHA}$ 的结合量大小依次为 $A>B>$ $C$; 在 $\left[{ }^{3} \mathrm{H}\right]-\mathrm{DHA}$ 浓度大于 $27.55 \mathrm{nmol} / \mathrm{L}$ 时, 重建 $\beta-A R$ 与 $\left[{ }^{3} \mathrm{H}\right]-\mathrm{DHA}$ 的结合量的大小依次为

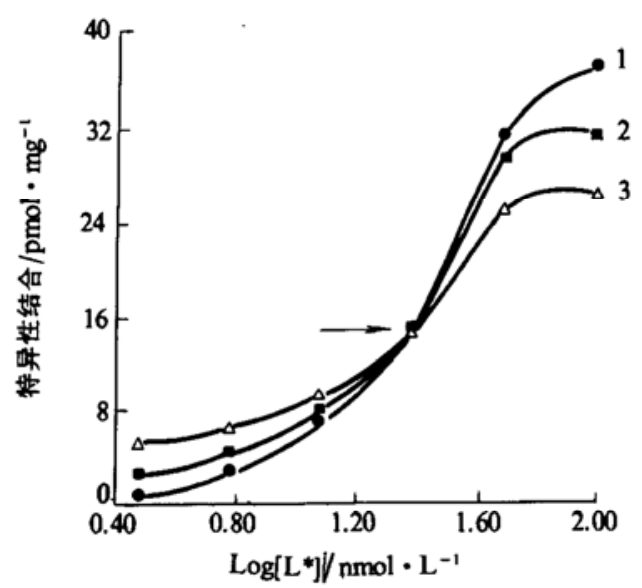

图 1 不同跨膜 $\mathrm{Ca}^{2+}$ 梯差脂蛋白体中 $\beta-\mathrm{AR}$ 与 $\left[{ }^{3} \mathrm{H}\right]-\mathrm{DHA}$ 结合的饱和曲线

“ $\rightarrow$ ”示其结合反应的拐点; 1-1 000:1 000, $2-1000: 10,3-1000: 1$

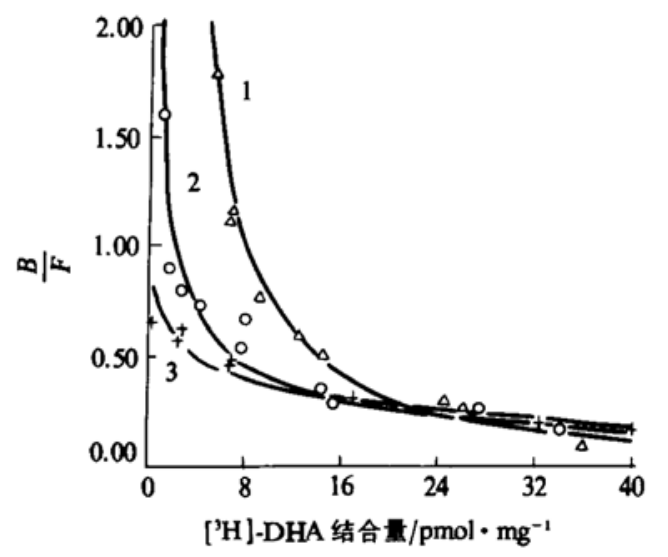

图 2 不同跨膜 $\mathrm{Ca}^{2+}$ 梯差脂蛋白体中 $\beta-\mathrm{AR}$ 与配

基结合的 Scatchard 曲线

$1-1000: 1,2-1000: 10,3-1000: 1000$

表 1 跨膜 $\mathrm{Ca}^{2+}$ 梯差对 $\mathrm{L} \cdot(\beta-\mathrm{AR})$ 脂蛋白体的受体结合容量 $(R)$ 和亲和力 $\left(K_{\mathrm{D}}\right)$ 的影响

\begin{tabular}{|c|c|c|c|c|}
\hline \multirow{2}{*}{$\begin{array}{l}\mathrm{L} \cdot(\beta-\mathrm{AR}) \\
\left(\left[\mathrm{Ca}^{2+}\right]_{\mathrm{o}}:\left[\mathrm{Ca}^{2+}\right]_{\mathrm{i}}\right)\end{array}$} & \multicolumn{2}{|c|}{ 高亲和结合 } & \multicolumn{2}{|c|}{ 低亲和结合 } \\
\hline & $\begin{array}{c}R_{1} \\
/ \mathrm{pmol} \cdot \mathrm{mg}^{-1}\end{array}$ & $\begin{array}{c}K_{\mathrm{D} 1} \\
/ \mathrm{nmol} \cdot \mathrm{L}^{-1}\end{array}$ & $\begin{array}{c}\quad R_{2} \\
/ \mathrm{pmol} \cdot \mathrm{mg}^{-1}\end{array}$ & $\begin{array}{c}K_{\mathrm{D} 2} \\
/ \mathrm{nmol} \cdot \mathrm{L}^{-1}\end{array}$ \\
\hline $1000: 1(A)$ & 10.5 & 3.02 & 45.2 & 129 \\
\hline $1000: 10(B)$ & 6.08 & 4.05 & 70.9 & 240 \\
\hline $1000: 1000(C)$ & 4.88 & 6.26 & 80.6 & 268 \\
\hline
\end{tabular}


$C>B>A$. 换言之, 随着脂酶体内 $\mathrm{Ca}^{2+}$ 浓度增加, $\mathrm{Ca}^{2+}$ 梯差减小, 高亲和力的结合位点结合 容量逐步减少, 即脂蛋白体 $A>B>C$, 出现所谓下行调节 (down-regulation); 而低亲和力的结 合位点结合容量则为 $C>B>A$, 即所谓上行调节 (up-regulation). 这种变化规律具有重要的 生理和病理学意义, 可能反映了机体在不同生理或病理条件下, 细胞对外界信息如儿茶酚胺类 物质刺激的一种生理性调节机制. 在信息跨膜转导的初始阶段, 儿茶酚胺类物质浓度较低时, 与生理状态相似的跨膜 $\mathrm{Ca}^{2+}$ 梯差有利于 $\beta-\mathrm{AR}$ 的高亲和力结合位点牢固地结合较多的配基以 启动 $\mathrm{cAMP}$ 信息跨膜转导体系的信息传递; 由于 $\mathrm{cAMP}$ 通过蛋白激酶 $\mathrm{A}$ 使 $\mathrm{Ca}^{2+}$ 通道磷酸化共 价修饰而开放 ${ }^{[2]}$, 使细胞外 $\mathrm{Ca}^{2+}$ 内流, 胞浆 $\left[\mathrm{Ca}^{2+}\right]$ 增加, 跨膜 $\mathrm{Ca}^{2+}$ 梯差减小 (类似于脂蛋白体 B), 此时 $\beta-A R$ 一方面结合较大量的配基以维持信息传递的继续. 另一方面, 由于这种结合的 亲和力较小, 结合相对较疏松, 易于解离以终止反应. 这可能提示在某些 $\mathrm{Ca}^{2+}$ 代谢案乱时, 跨 膜 $\mathrm{Ca}^{2+}$ 梯差发生病理改变 (如脂蛋白体 $\mathrm{C}$ ), 虽然儿茶酚胺类物质与 $\beta-\mathrm{AR}$ 低亲和位点的结合 量增大, 但由于亲和力太低, 细胞也难以产生对刺激物的应答性反应, 因而信息跨膜转导历程 不易启动.

综上所述, 本工作在重建的 $\beta-\mathrm{AR}$ 人工膜体系上所研究的跨膜 $\mathrm{Ca}^{2+}$ 梯差及其变化对 $\beta-\mathrm{AR}$ 配基结合功能影响的结果, 对于阐明一个合适的跨膜 $\mathrm{Ca}^{2+}$ 梯差及其一定范围内的变化对受体 牢固结合配基, 从而启动信息跨膜转导反应是必需的, 提供了较直接的实验证据. 同时还提示 当跨膜 $\mathrm{Ca}^{2+}$ 梯差发生较大变化时, 比如在某些病理条件下因 $\mathrm{Ca}^{2+}$ 代谢紊乱而导致的跨膜 $\mathrm{Ca}^{2+}$ 梯差变化, 对受体的配基结合功能将会产生不利影响, 从而导致机体对刺激的应答反应 调节失控.

\section{参考文献}

1 Williamson J R, Monck J R. Hormone effects on cellular $\mathrm{Ca}^{2+}$ fluxes. Annu Rev Physiol, 1989, 51: 109 124

2 Yang F Y, Huang Y G, Tu Y P. Divalent cation and lipid-protein interactions of biomembrane. Biosci Rep, 1993, 13; 143 $\sim 157$

3 Choi E J, Xia Z G, Villacres E C et al. The regulatory diversity of mammalian adenylate cyclase. Current Opinion in Cell Biology, 1993, 5: 369 373

4 Фye I, Sutherland E W. The effect of epinephrine and other agents on adenylyl cyclase in the cell membrane of avian erythrocyte. Biochim Biophys Acta, 1966, 127: 347 354

5 Caron M G, Srinivasan Y, Pitha J et al. Affinity chromatography of the $\beta$-adrenergic receptors. J Biol Chem, 1979, 254 : $2923 \sim 2927$

6 范高峰, 杨小毅, 黄有国. 北京鸭红细胞膜 $\beta$-肾上腺素能受体的纯化及其与 $\mathrm{Gs}$ 和 $\mathrm{AC}$ 在脂质体上的重建. 科学通报, 1995, 40(18): 1695 1698

7 Vauquelin G, Geynet P, Hanoune J et al. Isolation of adenylate cyclase-free, $\beta$-adrenergic receptors from turkey erythrocyte membrane by affinity chromatography. Proc Natl Acad Sci USA, 1977, 74: $3710 \sim 3714$

8 Kobilka B. Adrenergic receptors as models for G protein-coupled receptors. Annu Rev Neurosci, 1992, 15: 87 114 